Pacific

Journal of

Mathematics

HOWE DUALITY AND THE TRACE FORMULA

Zhengyu Mao and Stephen Rallis

Volume $191 \quad$ No. 2

December 1999 


\title{
HOWE DUALITY AND THE TRACE FORMULA
}

\author{
Zhengyu Mao and Stephen Rallis
}

\begin{abstract}
We state two families of trace formulas for the Howe duality. We prove the matching of orbital integrals for the trace formulas. The fundamental lemma (matching of orbital integrals for spherical functions) turns out to be the consequence of local Howe duality. We use the local Howe duality to prove the fundamental lemmas for another interesting family of trace formulas.
\end{abstract}

\section{Introduction.}

In $[\mathbf{H}]$, Howe conjectured a correspondence between the automorphic representations of $S O_{2 n+1}$ and these of $\tilde{S p_{n}}$. The local and global theory of the Howe correspondence has been established by the works of Furusawa $[\mathbf{F}]$, Howe [H2], Piatetski-Shapiro and Soudry [PS-S], Rallis [R], Waldspurger $[\mathbf{W}]$, etc. In this paper, we study the correspondence from a new angle, using the method of trace formula. We state two families of trace formulas that yield the Howe correspondence.

The local Howe duality play an essential role in our trace formula. Let $F_{v}$ be a non-Archimedean field with odd residue characteristics. We consider the Weil representation $\omega_{\psi}$ of the group $\tilde{S p_{n(2 n+1)}}\left(F_{v}\right)$ (the twofold cover of $\left.S p_{n(2 n+1)}\left(F_{v}\right)\right)$, which is determined by an additive character $\psi$ of $F_{v}$. We

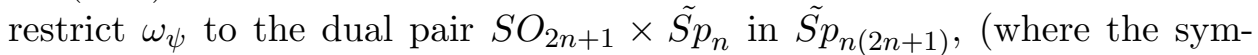
plectic form is given by the tensor product $Q \otimes A, Q$ being a symmetric nondegenerate bilinear form in $2 n+1$ variables, $A$ being an alternate nondegenerate bilinear form in $2 n$ variables). One version of the (local) Howe duality conjecture is: there exists a surjective homomorphism

$$
\lambda: \mathcal{H}\left(S O_{2 n+1} / / K\right) \rightarrow \mathcal{H}\left(\tilde{S p_{n}} / / K^{\prime}\right)
$$

between the spherical Hecke algebras of $\tilde{S p_{n}}$ and $S O_{2 n+1}$, so that when $\psi$ is of order 0 , for any $w \in \omega_{\psi}^{K \times K^{\prime}}$ (the space of $K \times K^{\prime}$ fixed vectors in $\omega_{\psi}$ ), we have

$$
\omega_{\psi}(f) w=\omega_{\psi}(\lambda(f)) w, f \in \mathcal{H}\left(S O_{2 n+1} / / K\right) .
$$

This conjecture is established in $[\mathbf{H 2}],[\mathbf{R}],[\mathbf{W}]$. 
In spectral terms, the identity (1) means that if any spherical irreducible representation $\pi_{1} \otimes \pi_{2}$ of $S O_{2 n+1} \times \tilde{S p_{n}}$ "occurs" in $\omega_{\psi}$, then

$$
\operatorname{Trace}\left(\pi_{2}(\lambda(f))\right)=\operatorname{Trace}\left(\pi_{1}(f)\right) .
$$

We will say $f$ and $\lambda(f)$ are spectrally matching functions. We observe here that from the viewpoint of the trace formula, the identity (1) in the case where $v$ is the spherical vector in $\omega_{\psi}$ is essentially the fundamental lemma, relating the orbital integrals for the matching Hecke functions.

We use the above observation to prove the fundamental lemma for another family of trace formulas. These trace formulas describe the lifting from automorphic representations of $\tilde{S L_{2}}$ to these of $S O_{2 n+1}$. Our proof avoids Jacquet's delicate argument in the $n=1$ case $[\mathbf{J}]$, relies rather on the existence of the Weil representation and the identity (1).

While the lifting considered here can be studied directly using the Weil representation, the trace formula approach yields extra information: An identity of distributions relating the representation of $S O_{2 n+1}$ (or $\tilde{S p_{n}}$ ) and its lifting. Such an identity has implications on the vanishing of certain linear functionals of the representations. In the case of Jacquet's formula $[\mathbf{J}]$, the identity has also interesting applications in the central $L$-values of cuspidal representations of $P G L_{2}$. The result in this paper suggests that the extra information may be obtained without much of the usual extra difficulties associated with the trace formula, namely, in the comparison of local orbital integrals.

\section{Two families of trace formulas.}

Let $F$ be a number field, $\mathbf{A}$ its adele ring. Let $Q(X)$ be a quadratic form on $F^{2 n+1}$ such that

$$
Q\left(x_{1}, \ldots, x_{2 n+1}\right)=2 x_{1} x_{2 n+1}+\cdots+2 x_{n} x_{n+2}+x_{n+1}^{2} .
$$

Denote by $\langle X, Y\rangle$ the associated bilinear form. Let $S O_{2 n+1}$ be the associated special orthogonal group. Let $S p_{n}$ be the symplectic group associated to the bilinear form $A$ with matrix $\left[\begin{array}{cc}I_{n} \\ -I_{n} & \end{array}\right]$. Let $\tilde{S p_{n}}$ be the twofold covering of $S p_{n}$. There is an embedding of $S O_{2 n+1} \times \tilde{S p_{n}}$ in $\tilde{S p_{n(2 n+1)}}$, (where the alternate form on $S p_{n(2 n+1)}$ is given by $\left.Q \otimes A\right)$, given by $(g, h) \rightarrow g \otimes h$. Under this embedding, we can regard $(g, h)$ as an element in $\tilde{S p_{n(2 n+1)}}$.

Fix a nontrivial additive character $\psi$ of $\mathbf{A} / F$. For such a $\psi$, there is a Weil representation $\omega_{\psi}$ of $\tilde{S p_{n}}$, acting on $\mathcal{S}\left(\mathbf{A}^{n}\right)$ the space of Schwartz functions on $\mathbf{A}^{n}$. We refer the reader to $[\mathbf{F}]$ for the definition of Weil representation. Let $\phi \in \mathcal{S}\left(\mathbf{A}^{n(2 n+1)}\right)$, we can then use the Weil representation 
$\omega_{\psi}$ of $\tilde{S p}_{n(2 n+1)}$ to define a theta function on $S O_{2 n+1} \times \tilde{S p_{n}}$ :

$$
\Theta_{\phi}^{\psi}(g, h)=\sum_{X \in F^{n(2 n+1)}} \omega_{\psi}(g, h) \phi(X), g \in S O_{2 n+1}, h \in \tilde{S} p_{n} .
$$

\subsection{Bessel model for $S O_{2 n+1}$ and Whittaker model.}

We introduce some subgroups of $S O_{2 n+1}$ and $\tilde{S p_{n}}$. Let $e_{1}, \ldots, e_{2 n+1}$ be the standard basis of $F^{2 n+1}$. Define a subgroup of $S O_{2 n+1}$ :

$$
\begin{aligned}
& D=\left\{d(a) \mid a \in G L_{1}, d(a) e_{n}=a e_{n}, d(a) e_{n+2}=a^{-1} e_{n+2},\right. \\
&\left.d(a) e_{i}=e_{i}, \quad i \neq n, n+2\right\} .
\end{aligned}
$$

Let $U_{l}$ be the group of unit upper triangular matrices in $G L_{l}$. Set

$$
\begin{gathered}
S^{\prime}=\left\{s(A, B)=\left[\begin{array}{ccc}
I_{n-1} & A & B \\
& I_{3} & A^{\prime} \\
& & I_{n-1}
\end{array}\right] \mid s(A, B) \in S O_{2 n+1}\right\}, \\
S=\left\{t(u)=\left[\begin{array}{ccc}
u & & \\
& I_{3} & \\
& & u^{*}
\end{array}\right] \in S O_{2 n+1} \mid u \in U_{n-1}\right\} .
\end{gathered}
$$

Let $R$ be the product of groups $D, S^{\prime}$ and $S$. Let $R^{\prime}$ be the subgroup of $S O_{2 n+1}$ consisting of elements that fixes $e_{1}, \ldots, e_{n-1}$ and $e_{n+1}$. Then $R^{\prime}$ is a subgroup of $R$. Let $N$ be the unit upper triangular subgroup of $S O_{2 n+1}$.

Let $V$ be the subgroup of $S p_{n}$ consisting of elements of the form $v(s)=$ $\left[\begin{array}{cc}I_{n} & s \\ & I_{n}\end{array}\right]$. Let $U_{n}^{\prime}$ be the subgroup of $S p_{n}$ with elements $t^{\prime}(u)=\left[\begin{array}{ll}u & \\ & u^{*}\end{array}\right]$ where $u \in U_{n}$. Let $N^{\prime}$ be the product of $U_{n}^{\prime}$ and $V$. Note the covering of $S p_{n}$ splits over $N^{\prime}$, thus we may consider $N^{\prime}$ as a subgroup of $\tilde{S p_{n}}$. For $n \in N^{\prime}$, we will use $\tilde{n}$ to denote the element $(n, 1)$ in $\tilde{S p_{n}}$.

Given an automorphic representation $\pi$ of $S O_{2 n+1}(\mathbf{A})$, we say it has a Bessel model with respect to $\psi$ if there is a $\phi$ in the space of $\pi$ such that:

$$
\int_{R(F) \backslash R(\mathbf{A})} \phi(r) \chi^{-1}(r) d r \neq 0 .
$$

Here $\chi$ is a character on $R$ with:

$$
\chi(t(u) s(A, B) d(a))=\psi\left(u_{1,2}+\cdots+u_{n-2, n-1}+A_{n-1,2}\right) .
$$

Note that $\chi$ is trivial on $R^{\prime}$.

Given an automorphic representation $\pi$ of $S O_{2 n+1}(\mathbf{A})$, we say it has a Whittaker model with respect to $\psi$ if there is a $\phi$ in the space of $\pi$ such that:

$$
\int_{N(F) \backslash N(\mathbf{A})} \phi(n) \theta\left(n^{-1}\right) d n \neq 0
$$

where $\theta(n)=\psi\left(n_{1,2}+\cdots+n_{n, n+1}\right)$ for $n \in N$. 
Given a genuine automorphic representation $\pi^{\prime}$ of $\tilde{S p_{n}}(\mathbf{A})$, we say it has a Whittaker model with respect to $\psi$ if there is a $\phi$ in the space of $\pi^{\prime}$ such that:

$$
\int_{N^{\prime}(F) \backslash N^{\prime}(\mathbf{A})} \phi(\tilde{n}) \theta^{\prime}\left(n^{-1}\right) \neq 0
$$

where $\theta^{\prime}\left(t^{\prime}(u) v(s)\right)=\psi\left(-u_{1,2}-\cdots-u_{n, n+1}+s_{n, n} / 2\right)$ for $v(s) \in V, u \in U_{n}$.

\subsection{The trace formulas.}

Let $C_{c}^{\infty}\left(\tilde{S p_{n}}(\mathbf{A})\right)$ be the set of genuine Schwartz functions on $\tilde{S p_{n}}(\mathbf{A})$. Given $f \in C_{c}^{\infty}\left(S O_{2 n+1}(\mathbf{A})\right)$ and a genuine function $f^{\prime} \in C_{c}^{\infty}\left(\tilde{S p_{n}}(\mathbf{A})\right)$, we can define kernel functions:

$$
\begin{gathered}
K_{f}(x, y)=\sum_{\gamma \in S O_{2 n+1}(F)} f\left(x^{-1} \gamma y\right), x, y \in S O_{2 n+1}(\mathbf{A}), \\
K_{f^{\prime}}(x, y)=\sum_{\gamma \in S p_{n}(F)} f\left(x^{-1} \cdot \tilde{\gamma} \cdot y\right), x, y \in \tilde{S p_{n}}(\mathbf{A}) .
\end{gathered}
$$

Note that the covering of $S p_{n}(\mathbf{A})$ splits over $S p_{n}(F)$, and we denote by $\tilde{\gamma}$ the element $(\gamma, 1)$ in $\tilde{S} p_{n}(\mathbf{A})$.

Our first claim is: There exists a map $(\delta, \epsilon)$ from $C_{c}^{\infty}\left(S O_{2 n+1}(\mathbf{A})\right)$ to $\mathcal{S}\left(\mathbf{A}^{n(2 n+1)}\right) \times C_{c}^{\infty}\left(\tilde{S p_{n}}(\mathbf{A})\right)$, such that we have an identity of distributions:

$$
I_{1}(f)=J_{1}(\delta(f), \epsilon(f))
$$

where

$$
\begin{gathered}
I_{1}(f)=\int_{R(F) \backslash R(\mathbf{A})} K_{f}(r, 1) \chi^{-1}(r) d r \\
J_{1}\left(\phi, f^{\prime}\right)=\int_{S p_{n}(F) \backslash \tilde{S p_{n}}(\mathbf{A})} \int_{N^{\prime}(F) \backslash N^{\prime}(\mathbf{A})} K_{f^{\prime}}(\tilde{n}, g) \Theta_{\phi}^{\psi}(1, g) \theta^{\prime}\left(n^{-1}\right) d n d g .
\end{gathered}
$$

The proof of this claim is indicated in Section 4. From the spectral decomposition of the identity (3), one expects to establish a correspondence between certain cuspidal automorphic representations of $S O_{2 n+1}(\mathbf{A})$ with a Bessel model and certain cuspidal automorphic representations of $\tilde{S p_{n}}(\mathbf{A})$ with a Whittaker model relative to the character $\psi$.

Our second claim is: There exists a map $\left(\delta^{\prime}, \epsilon^{\prime}\right)$ from $C_{c}^{\infty}\left(\tilde{S p_{n}}(\mathbf{A})\right)$ to $\mathcal{S}\left(\mathbf{A}^{n(2 n+1)}\right) \times C_{c}^{\infty}\left(S O_{2 n+1}(\mathbf{A})\right)$, such that we have an identity of distributions:

$$
I_{2}\left(\delta^{\prime}\left(f^{\prime}\right), \epsilon^{\prime}\left(f^{\prime}\right)\right)=J_{2}\left(f^{\prime}\right)
$$

where

$$
I_{2}(\phi, f)=\int_{S O_{2 n+1}(F) \backslash S O_{2 n+1}(\mathbf{A})} \int_{N(F) \backslash N(\mathbf{A})} K_{f}(g, n) \Theta_{\phi}^{\psi}(g, 1) \theta(n) d n d g,
$$




$$
J_{2}\left(f^{\prime}\right)=\int_{N^{\prime}(F) \backslash N^{\prime}(\mathbf{A})} K_{f^{\prime}}(\tilde{n}, 1) \theta^{\prime}\left(n^{-1}\right) d n .
$$

The proof of this claim is indicated in Section 4. From the spectral decomposition of the identity (4), one expects to establish a correspondence between certain cuspidal automorphic representations of $\tilde{S p_{n}}(\mathbf{A})$ with a Whittaker model relative to $\psi$ and certain cuspidal automorphic representations of $S O_{2 n+1}(\mathbf{A})$ with a Whittaker model relative to $\psi$.

The correspondences mentioned above are the Howe correspondence for the representations in question.

\section{Local orbital integrals.}

The definition of the distributions $I_{i}, J_{i}, i=1,2$ are motivated from the considerations in $[\mathbf{F}],[\mathbf{P S}-\mathbf{S}]$. These distributions can be unwinded into sums of orbital integrals.

Before stating the result of unwinding, we fix some notations. We will use two different models for the Weil representation $\omega_{\psi}$, associated to two polarizations of the symplectic space $F^{n(2 n+1)}$. The space $F^{n(2 n+1)}$ can be considered to be $Z=X \otimes Y$, where $X$ is the $2 n+1$ dimensional space equipped with the form $Q, Y$ is the $2 n$ dimensional space with the form $A$. Let $e_{i}$ be the standard basis of $X \cong F^{2 n+1}$ and $f_{i}$ be the standard basis of $Y \cong F^{2 n}$. Let $X^{+}$be the span of $e_{1}, \ldots e_{n}, Y^{+}$be the span of $f_{1}, \ldots, f_{n}$; they are two maximal isotropic space associated to $Q$ and $A$. Let $Z=Z^{+} \oplus Z^{-}$be a polarization of $Z$, then $\omega_{\psi}$ acts on $\mathcal{S}\left(Z^{+}\right)$.

One choice of the polarization is to let $Z^{+}=X \otimes Y^{+}$. In this case, an element in $Z^{+}$has the form $\oplus x_{i} \otimes f_{i}$; we denote such an element by $\left(x_{1}, \ldots, x_{n}\right)$.

The other choice of the polarization is to let $Z^{+}=X^{+} \otimes Y \oplus e_{n+1} \otimes Y^{+}$. For an element in $Z^{+}$, it has the form $\oplus e_{i} \otimes y_{i} \oplus e_{n+1} \otimes y^{\prime}$, where $y_{i} \in Y$ and $y^{\prime} \in Y^{+} \cong F^{n}$. We denote such an element by $\left(y_{1}, \ldots, y_{n}, y^{\prime}\right)$. We will use $f_{i}^{\prime}$ to denote the standard basis of $Y^{+} \cong F^{n}$.

For both polarization, for $f_{v}, f_{v}^{\prime}$ Schwartz functions of $S O_{2 n+1}\left(F_{v}\right)$ and $\tilde{S p_{n}}\left(F_{v}\right), \phi \in \mathcal{S}\left(Z^{+}\left(F_{v}\right)\right)$, define for $X \in Z^{+}\left(F_{v}\right)$

$$
\begin{aligned}
\omega_{\psi}\left(f_{v}\right) \phi_{v}(X) & =\int_{S O_{2 n+1}\left(F_{v}\right)} f_{v}(g) \omega_{\psi}(g, 1) \phi_{v}(X) d g, \\
\omega_{\psi}\left(f_{v}^{\prime}\right) \phi_{v}(X) & =\int_{\tilde{S p}_{n}\left(F_{v}\right)} f_{v}^{\prime}(g) \omega_{\psi}(1, g) \phi_{v}(X) d g .
\end{aligned}
$$

For $f$ a function on $S O_{2 n+1}\left(F_{v}\right)$, we let $\hat{f}(g)=f\left(g^{-1}\right)$. For $g \in \tilde{S p_{n}}\left(F_{v}\right)$, let $g^{\vee}=(\delta, 1) \cdot g \cdot(\delta, 1)^{-1}$, where $\delta=\left[\begin{array}{ll}-I_{n} & \\ & I_{n}\end{array}\right]$ lies in the similitude group $G S p_{n}$, (this automorphism is defined in [Mo-V-W, p. 90]). 
In this section, we prove:

Proposition 1. If $f=\otimes f_{v}, f^{\prime}=\otimes f_{v}^{\prime}$ and $\phi=\otimes \phi_{v}$ are products of local components, then:

$$
\begin{aligned}
I_{1}(f) & =\sum_{\gamma \in R(F) \backslash S O_{2 n+1}(F)} \prod_{v} I_{1, v}\left(\gamma_{v}, f_{v}\right), \\
J_{1}\left(\phi, f^{\prime}\right) & =\sum_{\gamma \in R(F) \backslash S O_{2 n+1}(F)} \prod_{v} J_{1, v}\left(\gamma_{v}, \omega_{\psi}\left(f_{v}^{\prime}\right) \phi_{v}\right), \\
I_{2}(\phi, f) & =\sum_{\gamma \in N^{\prime}(F) \backslash S p_{n}(F)} \prod_{v} I_{2, v}\left(\gamma_{v}, \omega_{\psi}\left(\hat{f}_{v}\right) \phi_{v}\right), \\
J_{2}\left(\phi, f^{\prime}\right) & =\sum_{\gamma \in N^{\prime}(F) \backslash S p_{n}(F)} \prod_{v} J_{2, v}\left(\gamma_{v}^{\vee}, f_{v}^{\prime}\right)
\end{aligned}
$$

where

$$
\begin{gathered}
I_{1, v}\left(\gamma_{v}, f_{v}\right)=\int_{R_{v}} f_{v}\left(r^{-1} \gamma_{v}\right) \chi^{-1}(r) d r \\
J_{1, v}\left(\gamma_{v}, \phi_{v}\right)=\int_{R_{v} / R_{v}^{\prime}} \omega_{\psi}\left(r^{-1} \gamma_{v}, 1\right) \phi_{v}\left(e_{1}, \ldots, e_{n-1}, e_{n+1}\right) \chi^{-1}(r) d r
\end{gathered}
$$

and

$$
\begin{gathered}
I_{2, v}\left(\gamma_{v}, \phi_{v}\right)=\int_{U_{n, v}} \omega_{\psi}\left(1, t^{\prime}(u)^{-1} \cdot \gamma_{v}\right) \phi_{v}\left(f_{n+1}, \ldots, f_{2 n},-f_{n}^{\prime}\right) \theta(t(u)) d u \\
J_{2, v}\left(\gamma_{v}^{\vee}, f_{v}^{\prime}\right)=\int_{N_{v}^{\prime}} f_{v}^{\prime}\left(\tilde{n}^{-1} \cdot \gamma_{v}^{\vee}\right) \theta^{\prime}\left(n^{-1}\right) d n .
\end{gathered}
$$

In $J_{1, v}\left(\gamma_{v}, \phi_{v}\right)$, we use the first polarization, while in $I_{2, v}\left(\gamma_{v}, \phi_{v}\right)$ we use the second polarization.

The proof of the proposition uses the computation that appears in $[\mathbf{F}]$. We will sketch the proof for the identity of $J_{1}\left(\phi, f^{\prime}\right)$. The following calculation is formal, however it could be justified using the argument similar to Prop. 1 in $[\mathbf{M}-\mathbf{R}]$.

We will use the following formulas for the Weil representation $\omega_{\psi}$. Using the polarization where $Z^{+}=X \otimes Y^{+}$:

$$
\begin{aligned}
\omega_{\psi}\left(g, t^{\prime}(u)\right) \phi\left(x_{1}, \ldots, x_{n}\right) & =\phi\left(g^{-1} x_{1} u, \ldots, g^{-1} x_{n} u\right) \\
\omega_{\psi}(1, \tilde{v}(S)) \phi\left(x_{1}, \ldots, x_{n}\right) & =\psi\left(\operatorname{Tr}\left(G r\left(x_{1}, \ldots, x_{n}\right) S\right) / 2\right) \phi\left(x_{1}, \ldots, x_{n}\right)
\end{aligned}
$$

where $G r\left(x_{1}, \ldots, x_{n}\right)=\left[\left\langle x_{i}, x_{j}\right\rangle\right]$ as an $n \times n$ matrix. 
Proof of the proposition. From the definition of $J_{1}\left(\phi, f^{\prime}\right)$ in $(2.2)$, we get it equals:

$$
\int_{S p_{n}(F) \backslash \tilde{S p_{n}}(\mathbf{A})} \int_{N^{\prime}(F) \backslash N^{\prime}(\mathbf{A})} \sum_{\gamma \in S p_{n}(F)} f^{\prime}\left(\tilde{n}^{-1} \cdot \tilde{\gamma} \cdot g\right) \Theta_{\phi}^{\psi}(1, g) \theta^{\prime}\left(n^{-1}\right) d n d g .
$$

Since $\Theta_{\phi}^{\psi}(1, \tilde{\gamma} \cdot g)=\Theta_{\phi}^{\psi}(g)$, we can unwind the above integral and get the following expression for $J_{1}\left(\phi, f^{\prime}\right)$ :

$$
\int_{\tilde{S p_{n}}(\mathbf{A})} \int_{N^{\prime}(F) \backslash N^{\prime}(\mathbf{A})} f^{\prime}\left(\tilde{n}^{-1} \cdot g\right) \Theta_{\phi}^{\psi}(1, g) \theta^{\prime}\left(n^{-1}\right) d n d g .
$$

Make a change of variable $g \rightarrow \tilde{n} \cdot g$. Using the notation in (6), we get

$$
J_{1}\left(\phi, f^{\prime}\right)=\int_{N^{\prime}(F) \backslash N^{\prime}(\mathbf{A})} \Theta_{\omega_{\psi}\left(f^{\prime}\right) \phi}^{\psi}(1, \tilde{n}) \theta^{\prime}\left(n^{-1}\right) d n
$$

which is

$$
\begin{aligned}
\left.\sum_{X \in F^{n(2 n+1)}} \int_{U_{n}(F) \backslash U_{n}(\mathbf{A})} \int_{V(F) \backslash V(\mathbf{A})} \omega_{\psi}(1, \tilde{v}) \omega_{\psi}\left(1, t^{\prime} \tilde{(u}\right)\right) \\
\cdot \omega_{\psi}\left(f^{\prime}\right) \phi(X) \theta^{\prime}\left(v^{-1} t^{\prime}(u)^{-1}\right) d u d v .
\end{aligned}
$$

For a fixed $X=\left(x_{1}, \ldots, x_{n}\right)$, the above integration has the form:

$$
\int_{V(F) \backslash V(\mathbf{A})} \Phi(X) \psi\left(\operatorname{Tr}\left(G r\left(x_{1}, \ldots, x_{n}\right) S\right) / 2-S_{n, n} / 2\right) d v(S)
$$

where $\Phi(X)$ equals:

$$
\left.\int_{U_{n}(F) \backslash U_{n}(\mathbf{A})} \omega_{\psi}\left(1, t^{\prime} \tilde{(u}\right)\right) \omega_{\psi}\left(f^{\prime}\right) \phi\left(x_{1}, \ldots, x_{n}\right) \theta^{\prime}\left(t^{\prime}(u)^{-1}\right) d u .
$$

The integration over $V$ is only nonzero for $X$ lying in the set:

$$
Z^{0}=\left\{\left(x_{1}, \ldots, x_{n}\right) \in Z^{+}(F) \mid\left\langle x_{i}, x_{j}\right\rangle=0 \text { except }\left\langle x_{n}, x_{n}\right\rangle=1\right\} .
$$

Over this set, the integration over $V$ gives $\Phi(X)$. Thus

$$
J_{1}\left(\phi, f^{\prime}\right)=\sum_{X \in Z^{0}} \int_{U_{n}(F) \backslash U_{n}(\mathbf{A})} \omega_{\psi}\left(1, \tilde{t^{\prime}} \tilde{(u)}\right) \omega_{\psi}\left(f^{\prime}\right) \phi\left(x_{1}, \ldots, x_{n}\right) \theta^{\prime}\left(t^{\prime}(u)^{-1}\right) d u .
$$

From Lemma 1 in $[\mathbf{F}]$, we can restrict the above sum to the subset where $x_{i}$ 's are linearly independent, (since $\Phi(X)=0$ otherwise). By Witts theorem, 
the expression (11) can be written as

$$
\begin{gathered}
\int_{U_{n}(F) \backslash U_{n}(\mathbf{A})} \sum_{\gamma \in R^{\prime}(F) \backslash S O_{2 n+1}(F)} \omega_{\psi}\left(1, t^{\prime}(u)\right) \omega_{\psi}\left(f^{\prime}\right) \\
\cdot \phi\left(\gamma^{-1} e_{1}, \ldots, \gamma^{-1} e_{n-1}, \gamma^{-1} e_{n+1}\right) \theta^{\prime}\left(t^{\prime}(u)^{-1}\right) d u \\
=\int_{U_{n}(F) \backslash U_{n}(\mathbf{A})} \sum_{\gamma \in R^{\prime}(F) \backslash S O_{2 n+1}(F)} \omega_{\psi}\left(\gamma, t^{\prime}(u)\right) \omega_{\psi}\left(f^{\prime}\right) \\
\cdot \phi\left(e_{1}, \ldots, e_{n-1}, e_{n+1}\right) \theta^{\prime}\left(t^{\prime}(u)^{-1}\right) d u .
\end{gathered}
$$

From the formula of the Weil representation, we see there is an isometry $\rho$ from $U_{n}$ to $R / R^{\prime}$, such that

$$
\left.\omega_{\psi}\left(1, t^{\prime} \tilde{(u}\right)\right) \phi\left(e_{1}, \ldots, e_{n-1}, e_{n+1}\right)=\omega_{\psi}\left(\rho(u)^{-1}, 1\right) \phi\left(e_{1}, \ldots, e_{n-1}, e_{n+1}\right)
$$

and $\theta^{\prime}\left(t^{\prime}(u)^{-1}\right)=\chi^{-1}(\rho(u))$. Thus $J_{1}\left(\phi, f^{\prime}\right)$ equals

$$
\begin{gathered}
\int_{\left(R / R^{\prime}(F)\right) \backslash\left(R / R^{\prime}(\mathbf{A})\right)} \sum_{R^{\prime}(F) \backslash S O_{2 n+1}(F)} \omega_{\psi}\left(r^{-1} \gamma, 1\right) \omega_{\psi}\left(f^{\prime}\right) \\
\cdot \phi\left(e_{1}, \ldots, e_{n-1}, e_{n+1}\right) \chi^{-1}(r) d r \\
=\int_{R(\mathbf{A}) / R^{\prime}(\mathbf{A})} \sum_{R(F) \backslash S O_{2 n+1}(F)} \omega_{\psi}\left(r^{-1} \gamma, 1\right) \omega_{\psi}\left(f^{\prime}\right) \\
\cdot \phi\left(e_{1}, \ldots, e_{n-1}, e_{n+1}\right) \chi^{-1}(r) d r .
\end{gathered}
$$

This identity implies our assertion on $J_{1}\left(\phi, f^{\prime}\right)$. The argument for $I_{2}(\phi, f)$ is similar, and we refer to $[\mathbf{F}]$ for the details. The unwinding for $I_{1}(f)$ and $J_{2}\left(f^{\prime}\right)$ is standard.

We note that the group $S p_{n}(F)$ is stable under the automorphism $g \rightarrow g^{\vee}$. Thus one may as well replace $\gamma_{v}^{\vee}$ by $\gamma_{v}$ in the equality (10). Our choice of the representative $\gamma_{v}^{\vee}$ however is more suitable for the comparison of local orbital integrals. (See below.)

\section{Proof of the claims: Fundamental lemma.}

From the Proposition 1, to show the claims in (2.2), we only need to prove the related identities between local orbital integrals. Fix a place $v$ of $F$. We will now drop the reference to $v$ in the notations.

If $v$ is a finite place with odd residue characteristic, let $\mathcal{O}$ be the ring of integers; we fix the Haar measures so that $S O_{2 n+1}(\mathcal{O})$ and $\tilde{S p_{n}}(\mathcal{O})$ have volume 1. Note that $S p_{n}(\mathcal{O})$ embeds in $\tilde{S p_{n}}(F)$ as a subgroup (as the covering splits over $S p_{n}(\mathcal{O})$ ), denoted $K^{\prime}$. Thus the maximal compact subgroup $\tilde{S p_{n}}(\mathcal{O})$ of $\tilde{S p_{n}}(F)$ is a direct product of $\{ \pm 1\}$ and $K^{\prime}$. The Hecke algebra of $\tilde{S p_{n}}(F)$ is the set $\mathcal{H}\left(\tilde{S p_{n}} / / K^{\prime}\right)$ of genuine compactly supported functions 
on $\tilde{S p_{n}}(F)$ which are bi-invariant under $K^{\prime}$. Similarly we define the Hecke algebra $\mathcal{H}\left(S O_{2 n+1} / / K\right)$ of $S O_{2 n+1}(F)$, where $K=S O_{2 n+1}(\mathcal{O})$. As in the introduction, there is an algebra homomorphism between the Hecke algebras of $S O_{2 n+1}(F)$ and $\tilde{S p_{n}}(F)$. We say two Hecke functions of $S O_{2 n+1}(F)$ and $\tilde{S p_{n}}(F)$ match if they correspond under the algebra homomorphism.

The following theorem yields our first claim:

\section{Theorem 1.}

(1) For all places $v$, given a function $f$, there exists functions $f^{\prime}$ and $\phi$, such that $I_{1}(\gamma, f)=J_{1}\left(\gamma, \omega_{\psi}\left(f^{\prime}\right) \phi\right)$ for all $\gamma \in S O_{2 n+1}(F)$.

(2) For almost all finite places $v$, if $f$ and $f^{\prime}$ are matching Hecke functions, and $\phi=\phi_{0}$ is the characteristic function of $Z^{+}(\mathcal{O})$, then $I_{1}(\gamma, f)=$ $J_{1}\left(\gamma, \omega_{\psi}\left(f^{\prime}\right) \phi\right)$ for all $\gamma \in S O_{2 n+1}(F)$.

The part 2 of the Theorem is the fundamental lemma for the trace formula (3). It is used to establish the functoriality of the correspondence. We will see that the fundamental lemma is essentially the Howe duality for unramified dual pairs, the identity (1) in introduction.

Proof of the Theorem. Since $\chi$ is trivial on $R^{\prime}$, we can write $I_{1}(\gamma, f)$ as

$$
\int_{R / R^{\prime}} \int_{R^{\prime}} f\left(h^{-1} r^{-1} \gamma\right) \chi^{-1}(r) d r d h .
$$

Define $\phi_{f}$ in $\mathcal{S}\left(Z^{+}(F)\right)$ by the identity

$$
\omega_{\psi}(g, 1) \phi_{f}\left(e_{1}, \ldots, e_{n-1}, e_{n+1}\right)=\int_{R^{\prime}} f\left(h^{-1} g\right) d h .
$$

Such a $\phi_{f}$ exists since $R^{\prime}$ is the fixator of $\left(e_{1}, \ldots, e_{n-1}, e_{n+1}\right)$. Thus

$$
I_{1}(\gamma, f)=\int_{R / R^{\prime}} \omega_{\psi}\left(r^{-1} \gamma, 1\right) \phi_{f}\left(e_{1}, \ldots, e_{n-1}, e_{n+1}\right) \chi^{-1}(r) d r .
$$

Compare with the expression for $J_{1}\left(\gamma, \omega_{\psi}\left(f^{\prime}\right) \phi\right)$, to prove part (1) of the theorem, we only need to show that: Given $f$, there exists $f^{\prime}, \phi$ such that $\omega_{\psi}\left(f^{\prime}\right) \phi=\phi_{f}$. This is clear.

For the proof of part (2), let $v$ be a finite place where $\psi$ has conductor $\mathcal{O}$ and the residue characteristic of $F$ is odd. This set of $v$ includes almost all finite places. Over this set of finite places $v$, the identity (1) holds for matching Hecke functions $f$ and $f^{\prime}$. Apply it to the case $w=\phi_{0}$, we see

$$
\omega_{\psi}(f) \phi_{0}=\omega_{\psi}\left(f^{\prime}\right) \phi_{0}
$$

for matching $f$ and $f^{\prime}$. Let $f_{0}$ and $f_{0}^{\prime}$ be the unit elements of the Hecke algebras of $S O_{2 n+1}$ and $\tilde{S p_{n}}$ respectively. Clearly

$$
\omega_{\psi}\left(f_{0}\right) \phi_{0}=\omega_{\psi}\left(f_{0}^{\prime}\right) \phi_{0}=\phi_{0} .
$$


If $g \in S O_{2 n+1}(F)$, let $g=$ ank be the Iwasawa decomposition of $g$, where $n \in N, k \in K$ and $a=\operatorname{diag}\left[a_{1}, a_{2}, \ldots, a_{1}^{-1}\right]$. We say $g$ is relevant if $\left|a_{i}\right| \leq\left|a_{i+1}\right| \leq 1$ for $i \leq n-2$. Note that $g$ being relevant is equivalent to $h g$ being relevant, for any $h \in R$.

Lemma 1. Let $g \in S O_{2 n+1}(F)$. With the above assumptions on the place $v$, if $g$ is not relevant, then $I_{1}(g, f)=J_{1}\left(g, \omega_{\psi}\left(f^{\prime}\right) \phi_{0}\right)=0$ for Hecke functions $f$ and $f^{\prime}$; if $g$ is relevant, then

$$
\omega_{\psi}(g, 1) \phi_{0}\left(e_{1}, \ldots, e_{n-1}, e_{n+1}\right)=\int_{R^{\prime}} f_{0}\left(h^{-1} g\right) d h .
$$

Assuming the Lemma, then the identity in part (2) of the Theorem holds for $\gamma$ not relevant. For $g$ relevant, for any Hecke function $f$ of $S O_{2 n+1}$, since $f\left(g^{-1}\right)=f(g)$, we have

$$
\begin{aligned}
\int_{R^{\prime}} f\left(h^{-1} g\right) d h & =\int_{R^{\prime}} \int_{S O_{2 n+1}(F)} f\left(j^{-1}\right) f_{0}\left(h^{-1} g j\right) d h d j \\
& =\int_{S O_{2 n+1}(F)} f(j) \omega_{\psi}(g j, 1) \phi_{0}\left(e_{1}, \ldots, e_{n-1}, e_{n+1}\right) d j \\
& =\omega_{\psi}(g, 1) \omega_{\psi}(f) \phi_{0}\left(e_{1}, \ldots, e_{n-1}, e_{n+1}\right) .
\end{aligned}
$$

Thus if $\gamma$ is relevant, we can take $\phi_{f}$ in the expression (12) to be $\omega_{\psi}(f) \phi_{0}$. The assertion (2) for the relevant $\gamma$ follows from the identity (13).

Proof of the Lemma. If $g$ is not relevant, we show the vanishing of $I_{1}(g, f)$ and $J_{1}\left(g, \omega_{\psi}\left(f^{\prime}\right) \phi\right)$. There is $i<n-1$ with $\left|a_{i}\right|>\left|a_{i+1}\right|$ or $\left|a_{n-1}\right|>1$. We may as well assume $g$ is in the diagonal form. There is an $x$ with $\psi(x) \neq 1$ and $\left|\frac{a_{i+1}}{a_{i}} x\right| \leq 1$ or $\left|a_{n-1}^{-1} x\right| \leq 1$. Let $T$ be an element in $N$ with the $(i, i+1)$ th (or $(n-1, n+1)$ th) entry $x$, and other $T_{i, j}=0$ if $i \leq n, i \neq j$. Then

$$
I_{1}(g, f)=\psi(x) I_{1}(T g, f)=\psi(x) I_{1}\left(g T^{\prime}, f\right)
$$

with $T^{\prime} \in S O_{2 n+1}(\mathcal{O})$. Thus $I_{1}(g, f)=\psi(x) I_{1}(g, f)$ equals 0 . Same argument shows $J_{1}\left(g, \omega_{\psi}\left(f^{\prime}\right) \phi_{0}\right)=0$ when $g$ is not relevant.

To prove the identity (14), let $F_{1}(g)$ and $F_{2}(g)$ be the LHS and RHS of the equation. Since $F_{i}(h g k)=F_{i}(g)$ for $i=1,2, h \in R^{\prime}, k \in S O_{2 n+1}(\mathcal{O})$, we only need to show the identity for $g=T d$, with $T \in N$ and $T_{i, n}=T_{i, n+2}=0$, $i<n$, and $d$ being in diagonal form and relevant, and $\left|a_{n}\right|=1$. For such a $g$, a simple computation shows that $F_{i}(g)$ equal 1 when $T \in N(\mathcal{O})$ and $\left|a_{i}\right|=1$ for all $i$, they equal 0 otherwise.

Remark. We ask the question whether the validity of the fundamental lemma implies the existence of Howe duality. That is, can we prove a priori that if for all $\gamma \in S O_{2 n+1}(F), I_{1}(\gamma, f)=J_{1}\left(\gamma, \omega_{\psi}\left(f^{\prime}\right) \phi_{0}\right)$ for matching functions $f$ and $f^{\prime}$, then the identity (13) is valid? In particular, we ask for any 
$\tilde{\phi}$ being $K \times K^{\prime}$ invariant, whether the vanishing of

$$
\int_{R / R^{\prime}} \omega_{\psi}\left(r^{-1} \gamma, 1\right) \tilde{\phi}\left(e_{1}, \ldots, e_{n-1}, e_{n+1}\right) \chi^{-1}(r) d r=0
$$

for all $\gamma \in S O_{2 n+1}(F)$ implies that $\tilde{\phi} \equiv 0$. An affirmative answer would imply that Theorem 1 (2) yields the identity (13).

We now consider the second claim in (2.2). Its proof is similar to that of the first claim. We will state the results and give a sketch of the proof:

\section{Theorem 2.}

(1) For all places $v$, given a function $f^{\prime}$, there exists functions $f$ and $\phi$, such that $J_{2}\left(\gamma^{\vee}, f^{\prime}\right)=I_{2}\left(\gamma, \omega_{\psi}(\hat{f}) \phi\right)$ for all $\gamma \in \tilde{S} p_{n}(F)$.

(2) For almost all finite places $v$, if $f$ and $f^{\prime}$ are matching Hecke functions, and $\phi=\phi_{0}$ is the characteristic function of $Z^{+}(\mathcal{O})$, then $J_{2}\left(\gamma^{\vee}, f^{\prime}\right)=$ $I_{2}\left(\gamma, \omega_{\psi}(\hat{f}) \phi\right)$ for all $\gamma \in \tilde{S p_{n}}(F)$.

Proof. We can write $J_{2}\left(\gamma^{\vee}, f^{\prime}\right)$ as

$$
\int_{V} \int_{U_{n}} f^{\prime}\left(v \tilde{(s)}{ }^{-1} t^{\prime}(u)^{-1} \gamma^{\vee}\right) \theta^{\prime}\left(v(s)^{-1} t^{\prime}(u)^{-1}\right) d u d v(s) .
$$

Since $t^{\prime} \tilde{(u)^{\vee}}=t^{\prime}(u)$ and $\theta^{\prime}\left(t^{\prime}(u)^{-1}\right)=\theta(t(u))$, we get

$$
\left.\int_{V} \int_{U_{n}} f^{\prime}(v \tilde{(s)})^{-1} t^{\prime} \tilde{(u)^{\vee-1}} \gamma^{\vee}\right) \theta^{\prime}\left(v(s)^{-1}\right) \theta(t(u)) d u d v(s) .
$$

Since the group $V$ is stable under the automorphism $g \rightarrow g^{\vee}$, and $\tilde{v(s)} \tilde{\vee}^{\vee}=$ $\tilde{v(s)^{-1}}$, there is a function $\phi_{f^{\prime}}$ on $Z^{+}(F)$ with

$$
\omega_{\psi}(1, g) \phi_{f^{\prime}}\left(f_{n+1}, \ldots, f_{2 n},-f_{n}^{\prime}\right)=\int_{V} f^{\prime}\left(v(s)^{-1} g^{\vee}\right) \theta^{\prime}\left(v(s)^{-1}\right) d v(s) .
$$

Thus $J_{2}\left(\gamma^{\vee}, f^{\prime}\right)$ equals:

$$
\int_{U_{n}} \omega_{\psi}\left(1, t^{\prime}(u)^{-1} \gamma\right) \phi_{f^{\prime}}\left(f_{n+1}, \ldots, f_{2 n},-f_{n}^{\prime}\right) \theta(t(u)) d u .
$$

Given $f^{\prime}$, we choose $\phi$ and $f$ such that $\omega_{\psi}(\hat{f}) \phi=\phi_{f^{\prime}}$; such a pair satisfies the condition in part (1) of the Theorem.

We now show part (2) of the Theorem. Make the assumption on the place $v$ as in the proof of part (2) of Theorem 1. For $g \in \tilde{S p_{n}}(F)$, let $g=a n k$ be the Iwasawa decomposition, where $n \in N^{\prime}, k \in K^{\prime}$ and $a=$ $\left(\operatorname{diag}\left[a_{1}, a_{2}, \ldots, a_{n}^{-1}\right], \zeta\right), \zeta \in\{ \pm 1\}$. We say $g$ is relevant if $\left|a_{i}\right| \leq\left|a_{i+1}\right|$ for $1 \leq i<n$. 
Lemma 2. If $g$ is not relevant, then $I_{2}\left(g, \omega_{\psi}(\hat{f}) \phi_{0}\right)=J_{2}\left(g^{\vee}, f^{\prime}\right)=0$ when $f$ and $f^{\prime}$ are Hecke functions. When $g$ is relevant,

$$
\omega_{\psi}(1, g) \phi_{0}\left(f_{n+1}, \ldots, f_{2 n},-f_{n}^{\prime}\right)=\int_{V} f_{0}^{\prime}\left(\tilde{v}^{-1} g^{\vee}\right) \theta^{\prime}\left(v^{-1}\right) d v .
$$

We skip the proof of the Lemma, which is similar to that of Lemma 1, and uses the fact that $K^{\prime}$ is stable under the automorphism $g \rightarrow g^{\vee}$. From the Lemma, the identity in part (2) holds for $\gamma$ not relevant. When $\gamma$ is relevant, from (15), $J_{2}\left(\gamma^{\vee}, f^{\prime}\right)$ equals:

$$
\iint_{V} \int_{U_{n}} f_{0}^{\prime}\left(v \tilde{(s)}{ }^{-1} t^{\prime} \tilde{(u)^{\vee-1}} \gamma^{\vee} g^{\vee}\right) f^{\prime}\left(g^{\vee-1}\right) \theta^{\prime}\left(v(s)^{-1}\right) \theta(t(u)) d u d v(s) d g .
$$

From the Lemma and the fact $f^{\prime}\left(g^{\vee-1}\right)=f^{\prime}(g)([\mathbf{M o}-\mathbf{V}-\mathbf{W}$, p. 92] $)$, we get

$$
\begin{aligned}
& \iint_{U_{n}} f^{\prime}(g) \omega_{\psi}\left(1, t^{\prime}(u)^{-1} \gamma g\right) \phi_{0}\left(f_{n+1}, \ldots, f_{2 n},-f_{n}^{\prime}\right) \theta(t(u)) d u d g \\
= & \int_{U_{n}} \omega_{\psi}\left(1, t^{\prime}(u)^{-1} \gamma\right) \omega_{\psi}\left(f^{\prime}\right) \phi_{0}\left(f_{n+1}, \ldots, f_{2 n},-f_{n}^{\prime}\right) \theta(t(u)) d u .
\end{aligned}
$$

Once again from identity (1), we get the identity (13), and the Theorem follows from the identity (13) and the fact $f(g)=\hat{f}(g)$ when $f \in$ $\mathcal{H}\left(S O_{2 n+1} / / K\right)$.

We note that the identities in Lemma 1 and 2 are not true for $g$ not relevant. For example in Lemma 2 if $g$ is in diagonal form, with $\left|a_{n}\right|=1$ and $\left|a_{i}\right|>1, i \neq n$, then the RHS of (16) equals 0 while the LHS is nonzero.

\section{The lifting from $\tilde{S L_{2}}$ to $S O_{2 n+1}$.}

In $[\mathbf{M}-\mathbf{R}]$, we introduced a family of trace formula that describes the liftings of automorphic representations of $S L_{2}$ and $\tilde{S L_{2}}$. The argument in [M-R] suggests the following trace formula: Given a genuine function $f^{\prime}$ in $C_{c}^{\infty}\left(\tilde{S L_{2}}(\mathbf{A})\right)$, there is a function $\epsilon\left(f^{\prime}\right) \in C_{c}^{\infty}\left(S O_{2 n+1}(\mathbf{A})\right)$, such that $I\left(\epsilon\left(f^{\prime}\right)\right)=J\left(f^{\prime}\right)$ where

$$
I(f)=\int_{H_{n+1}(F) \backslash H_{n+1}(\mathbf{A})} \int_{N^{\prime \prime}(F) \backslash N^{\prime \prime}(\mathbf{A})} K_{f}(h, n) \theta^{\prime \prime}(n) d n d h
$$

and

$$
J\left(f^{\prime}\right)=\int_{N^{\prime} \times N^{\prime}(F) \backslash N^{\prime} \times N^{\prime}(\mathbf{A})} K_{f^{\prime}}\left(\tilde{n_{1}}, \tilde{n_{2}}\right) \theta^{\prime}\left(n_{1} n_{2}^{-1}\right) d n_{1} d n_{2} .
$$

Here we kept the notations in the previous sections, the group $H_{n+1}$ is the fixator of $e_{n+1}$ in $S O_{2 n+1}(F)$; it is isomorphic to $S O_{2 n}$; the group $N^{\prime \prime}$ 
consists of elements of the form

$$
c(X)=\left[\begin{array}{ccc}
1 & X & -\langle X, X\rangle / 2 \\
& I_{2 n-1} & X^{\prime} \\
& & 1
\end{array}\right]
$$

where $X \in F^{2 n-1}$. Let $e_{i}^{\prime}$ be the standard basis of $F^{2 n-1}$; let $\theta^{\prime \prime}(c(X))=$ $\psi\left(\left\langle X, e_{n}^{\prime}\right\rangle\right)$. For a vector $X$, we will let $X_{i}$ be the $i$-th coordinate of $X$. Thus $\left\langle X, e_{n}^{\prime}\right\rangle=X_{n}$.

Observe that when $n=1$, our trace formula is just the relative trace formula proved in $[\mathbf{J}]$. From the trace formula, we expect to get a lifting from the cuspidal representations of $\tilde{S L_{2}}$ to the automorphic representation of $S O_{2 n+1}$ with a nonvanishing $S O_{2 n}$-period and a degenerate Whittaker model with respect to $\theta^{\prime \prime-1}$ on $N^{\prime \prime}$.

The purpose of this section is to unwind the integrals $I(f)$ and $J\left(f^{\prime}\right)$ as in Section 3, and prove the fundamental lemma for the trace formulas. The proof uses the formula (1) and the formulas of the Weil representation.

\subsection{Local orbital integrals.}

We prove a similar result as in Proposition 1.

Given $f \in C_{c}^{\infty}\left(S O_{2 n+1}(\mathbf{A})\right)$, there is a function $\phi_{f} \in \mathcal{S}\left(\mathbf{A}^{2 n+1}\right)$ satisfying:

$$
\omega_{\psi^{-1}}(g, 1) \phi_{f}\left(e_{n+1}\right)=\int_{H_{n+1}(\mathbf{A})} f\left(h^{-1} g\right) d h .
$$

Here we are using the polarization of first type, (see $\S 4$ ). Thus $I(f)$ equals:

$$
\begin{aligned}
& \sum_{\gamma \in H_{n+1}(F) \backslash S O_{2 n+1}(F)} \int_{H_{n+1}(\mathbf{A})} \int_{\mathbf{A}^{2 n-1} / F^{2 n-1}} f\left(h^{-1} \gamma c(X)\right) \psi\left(X_{n}\right) d X d h \\
= & \sum_{\gamma \in H_{n+1}(F) \backslash S O_{2 n+1}(F)} \int_{\mathbf{A}^{2 n-1} / F^{2 n-1}} \omega_{\psi^{-1}}(\gamma c(X), 1) \phi_{f}\left(e_{n+1}\right) \psi\left(X_{n}\right) d X \\
= & \sum_{\langle Y, Y\rangle=1, Y \in F^{2 n+1}} \int_{\mathbf{A}^{2 n-1} / F^{2 n-1}} \phi_{f}\left(c(X)^{-1} Y\right) \psi\left(X_{n}\right) d X .
\end{aligned}
$$

In the last step, we applied the Witts theorem and the formula for Weil representation. Separate the above sum into two parts, according to whether $Y_{2 n+1}$ equals 0 or not. If $Y$ is such that $Y_{2 n+1}=0$ and there is an $i \neq n+1,1$ with $Y_{i} \neq 0$, then there exists $X^{0}$ with $\psi\left(X_{n}^{0}\right) \neq 1$ such that $c\left(X^{0}\right)^{-1} Y=Y$. For such a $Y$, the integration over $X$ then gives value 0 . Thus we can write the above sum as $I^{+}(f)+I^{-}(f)+I^{\prime}(f)$, where $I^{\prime}(f)$ is the above sum with condition $Y_{2 n+1} \neq 0$ and

$$
I^{ \pm}(f)=\sum_{y \in F} \int_{\mathbf{A}^{2 n-1} / F^{2 n-1}} \phi_{f}\left(C(X)^{-1} Y^{ \pm}(y)\right) \psi\left(X_{n}\right) d X
$$


where $Y^{ \pm}(y)$ is the vector with $Y^{ \pm}(y)_{n+1}= \pm 1, Y^{ \pm}(y)_{1}= \pm y$ and $Y^{ \pm}(y)_{i}=$ 0 for other $i$ 's. Assuming the measure is so chosen that the volume of $\mathbf{A} / F$ is 1 . The integral $I^{ \pm}(f)$ unwinds into

$$
\int_{X_{n} \in \mathbf{A}} \phi_{f}\left(Y^{ \pm}\left(X_{n}\right)\right) \psi\left(X_{n}\right) d X_{n}
$$

Meanwhile the integral $I^{\prime}(f)$ equals

$$
\begin{aligned}
& \sum_{Y_{2 n+1} \in F^{\times},\langle Y, Y\rangle=1} \int_{X \in \mathbf{A}^{2 n-1} / F^{2 n-1}} \phi_{f} \\
& \cdot\left(Y_{1}^{\prime}, Y_{2}-Y_{2 n+1} X_{2 n-1}, \ldots, Y_{2 n}-Y_{2 n+1} X_{1}, Y_{2 n+1}\right) \psi\left(X_{n}\right) d X
\end{aligned}
$$

where $Y_{1}^{\prime}$ is the only number which gives the vector length 1 . Make changes of variables $X_{i} \rightarrow-Y_{2 n+1}^{-1}\left(X_{i}+Y_{2 n+1-i}\right)$, the integral unwinds into:

$$
\begin{aligned}
\sum_{Y_{2 n+1} \in F^{\times}} \int_{X \in \mathbf{A}^{2 n-1}} \phi_{f} \\
\cdot\left(\frac{1-\langle X, X\rangle}{2 Y_{2 n+1}}, X_{2 n-1}, \ldots, X_{1}, Y_{2 n+1}\right) \psi\left(-X_{n} / Y_{2 n+1}\right) d X_{n} .
\end{aligned}
$$

From the above discussion, we get:

Proposition 2. If $f=\otimes f_{v}$, then there exists $\phi_{f}=\otimes \phi_{f, v}$, so that

$$
I(f)=\sum_{a \in F^{\times}} \prod_{v} I_{v}\left(a_{v}, f_{v}\right)+\prod_{v} I_{v}^{+}\left(f_{v}\right)+\prod_{v} I_{v}^{-}\left(f_{v}\right)
$$

where

$$
\begin{gathered}
I_{v}^{ \pm}\left(f_{v}\right)=\int_{F_{v}} \phi_{f, v}\left(Y^{ \pm}(x)\right) \psi(x) d x \\
I_{v}\left(a, f_{v}\right)=\int_{F_{v}^{2 n-1}} \phi_{f, v}\left(\frac{1-\langle X, X\rangle}{2 a}, X, a\right) \psi\left(-X_{n} / a\right) d X .
\end{gathered}
$$

The unwinding for $J\left(f^{\prime}\right)$ is standard; we state the result here, (see $[\mathbf{J}]$ ).

Proposition 3. If $f^{\prime}=\otimes f_{v}^{\prime}$, then

$$
J\left(f^{\prime}\right)=\sum_{a \in F^{\times}} \prod J_{v}\left(a_{v}, f^{\prime}\right)+\prod J_{v}^{+}\left(f^{\prime}\right)+\prod J_{v}^{-}\left(f^{\prime}\right)
$$

where

$$
\begin{gathered}
J_{v}^{ \pm}\left(f_{v}^{\prime}\right)=\int_{F_{v}} f_{v}^{\prime}(( \pm v(x), 1)) \psi(-x / 2) d x \\
J_{v}\left(a, f_{v}^{\prime}\right)=\int_{F_{v}^{2}} f^{\prime}\left(v(x) \cdot w^{\vee} \cdot m(a)^{\vee} \cdot v(y)\right) \psi(-(x+y) / 2) d x d y .
\end{gathered}
$$


Here

$$
\begin{gathered}
v(x)=\left(\left[\begin{array}{ll}
1 & x \\
& 1
\end{array}\right], 1\right), \quad w=\left(\left[\begin{array}{ll} 
& 1 \\
-1
\end{array}\right], 1\right) \\
\text { and } m(a)=\left(\left[\begin{array}{ll}
a & \\
& a^{-1}
\end{array}\right], 1\right) .
\end{gathered}
$$

\subsection{The fundamental lemma.}

Let $v$ be a finite place where $\psi$ has conductor $\mathcal{O}$ and the residue characteristic is odd. We study the orbital integrals over the field $F_{v}$. We will drop the references for $v$ in the notations.

Let $f$ and $f^{\prime}$ be Hecke functions as in Section 4. The concept of matching functions is also defined in the current situation. For matching Hecke functions, the identity (1) still holds. Also (13) holds. We prove:

Theorem 3. If $f$ and $f^{\prime}$ are matching Hecke functions, then $I^{ \pm}(f)=$ $J^{ \pm}\left(f^{\prime}\right)$, and $I(a, f)=|a|^{n+1 / 2} \gamma\left(a, \psi^{-1}\right) J\left(a, f^{\prime}\right), a \in F^{\times}$.

This theorem is the fundamental lemma. The factor $|a|^{n+1 / 2} \gamma\left(a, \psi^{-1}\right)$ is called the transfer factor. The Weil constant $\gamma(a, \psi)$ is defined by:

$$
\int \hat{\Phi}(x) \psi\left(a x^{2} / 2\right) d x=|a|^{-1 / 2} \gamma(a, \psi) \int \Phi(x) \psi\left(-a^{-1} x^{2} / 2\right) d x
$$

where $\hat{\Phi}$ is the Fourier transform of $\Phi$

$$
\hat{\Phi}(x)=\int \Phi(y) \psi(x y) d y .
$$

In the $n=1$ case, the theorem is proved in $[\mathbf{J}]$ using relation between relative Kloosterman sum and Salié sum. The $n=2$ case was proved by FlickerMars [Fl-Ma] (for the unit Hecke functions $f_{0}$ and $f_{0}^{\prime}$ ) and Zinoviev $[\mathbf{Z}]$ (for general Hecke functions). Our method of proof is different from theirs, and gives a considerably shorter proof for the general case. We note also that our transfer factor is different from the one in $[\mathbf{J}]$, that is because we define our local orbital integrals differently.

The Theorem follows from a couple of Lemmas.

Lemma 3. Let $f$ be a Hecke function of $S O_{2 n+1}(F)$ and $\phi_{0}$ be the characteristic function of $\mathcal{O}^{2 n+1}$, then

$$
\omega_{\psi^{-1}}(g, 1) \omega_{\psi^{-1}}(f) \phi_{0}\left(e_{n+1}\right)=\int_{H_{n+1}(F)} f\left(h^{-1} g\right) d h .
$$

Proof. Similar to the argument in Theorem 1, we only need to show the above identity for $f=f_{0}$. Let $K=S O_{2 n+1}(\mathcal{O})$. Then the identity is equivalent to the following: For $g \in S O_{2 n+1}(F), g e_{n+1} \in \mathcal{O}^{2 n+1}$ if and only if $g \in K H_{n+1}$. Observe that any vector $X=g e_{n+1}$ satisfies $\langle X, X\rangle=1$. Thus $K$ acts transitively on the intersection of $\mathcal{O}^{2 n+1}$ and the $S O_{2 n+1}(F)$ orbit 
of $e_{n+1}$. Under the assumption of 1 ), there is a $k \in K$ with $g e_{n+1}=k e_{n+1}$. Thus $k^{-1} g \in H_{n+1}$ and $g \in K H_{n+1}$.

From the Lemma, we can let $\phi_{f}$ in the definition of $I(f)$ and $I(a, f)$ to be $\omega_{\psi^{-1}}(f) \phi_{0}$.

Lemma 4. If $f^{\prime}$ is a Hecke function of $\tilde{S L_{2}}(F)$, then

$$
\int_{F} \omega_{\psi^{-1}}(1, g) \omega_{\psi^{-1}}\left(f^{\prime}\right) \phi_{0}\left(Y^{+}(x)\right) \psi(x) d x=\int_{F} f^{\prime}\left(v(x) \cdot g^{\vee}\right) \psi(-x / 2) d x .
$$

Proof. As in the argument of Theorem 2, we only need to show the identity for $f_{0}^{\prime}$. Denote by $F_{1}(g)$ and $F_{2}(g)$ the LHS and RHS of the equation (20) when $f^{\prime}=f_{0}^{\prime}$. Observe that both $F_{1}(g)$ and $F_{2}(g)$ are genuine functions on $\tilde{S L_{2}}$ and satisfy:

$$
F_{i}(v(s) g k)=\psi(-s / 2) F_{i}(g), s \in F, k \in S L_{2}(\mathcal{O}) .
$$

Thus we only need to show $F_{1}(m(a))=F_{2}(m(a))$. It is clear that $F_{2}(m(a))=$ 1 when $a \in \mathcal{O}^{\times}$and 0 otherwise. While

$$
F_{1}(m(a))=\int_{a, a x \in \mathcal{O}} \psi(x) d x
$$

which clearly equals 1 when $a \in \mathcal{O}^{\times}$and 0 otherwise. We have shown the identity for $g=m(a)$ and the Lemma.

Apply Lemma 4 to the case $g=\left( \pm I_{2}, 1\right)$. Then $g^{\vee}=g$, we see

$$
J^{ \pm}\left(f^{\prime}\right)=\int_{F} \omega_{\psi^{-1}}\left(f^{\prime}\right) \phi_{0}\left(Y^{ \pm}(x)\right) \psi(x) d x .
$$

The fact $I^{ \pm}(f)=J^{ \pm}\left(f^{\prime}\right)$ follows immediately from Lemma 3 and the identity (13).

Fix now $a \in F^{\times}$. From the Lemma and the fact $v(-y)^{\vee}=v(y)$,

$$
J\left(a, f^{\prime}\right)=\int_{F} F_{1}(w \cdot m(a) \cdot v(-y)) \psi(-y / 2) d y .
$$

Recall the action of $w$ on the space of Weil representation is essentially the Fourier transform. Using the formulas in $[\mathbf{F}]$, we see $F_{1}(w \cdot m(a) \cdot v(-y))$ equals:

$$
\int_{F} \omega_{\psi^{-1}}(1, w \cdot m(a) \cdot v(-y)) \omega_{\psi^{-1}}\left(f^{\prime}\right) \phi_{0}\left(Y^{+}(x)\right) \psi(x) d x
$$

which is $\gamma\left(a, \psi^{-1}\right)^{-1}|a|^{n+1 / 2}$ times

$$
\int_{F} \int_{F^{2 n+1}} \omega_{\psi^{-1}}\left(f^{\prime}\right) \phi_{0}(a Y) \psi^{-1}\left(\left\langle Y, Y^{+}(x)\right\rangle\right) \psi(x) \psi(\langle a Y, a Y\rangle y / 2) d x d Y
$$


Note that $\left\langle Y, Y^{+}(x)\right\rangle=Y_{n+1}+x Y_{2 n+1}$. The integral over $Y_{2 n+1}$ and $x$ has the form

$$
\int_{F} \int_{F} \phi\left(Y_{2 n+1}\right) \psi\left(x-x Y_{2 n+1}+a^{2} y Y_{2 n+1} Y_{1}\right) d x d Y_{2 n+1} .
$$

Using the fact that $\hat{\hat{\phi}}(x)=\phi(-x)$, the above integral equals $\phi(1) \psi\left(a^{2} y Y_{1}\right)$. Integrate over $x$ and $Y_{2 n+1}$; use $X$ to denote the vector $\left(Y_{2}, \ldots, Y_{2 n}\right),(21)$ becomes:

$$
\begin{aligned}
\int_{X \in F^{2 n-1}, Y_{1} \in F} & \omega_{\psi^{-1}}\left(f^{\prime}\right) \\
\cdot & \phi_{0}\left(a Y_{1}, a X, a\right) \psi\left(-X_{n}\right) \psi\left(a^{2} y Y_{1}+\langle a X, a X\rangle y / 2\right) d Y_{1} d X .
\end{aligned}
$$

Thus $J\left(a, f^{\prime}\right)$ equals $\gamma\left(a, \psi^{-1}\right)^{-1}|a|^{n+1 / 2}$ times:

$$
\begin{aligned}
& \int_{X \in F^{2 n-1}, Y_{1}, y \in F} \omega_{\psi^{-1}}\left(f^{\prime}\right) \\
& \quad \cdot \phi_{0}\left(a Y_{1}, a X, a\right) \psi\left(-X_{n}\right) \psi\left(a^{2} y Y_{1}+\langle a X, a X\rangle y / 2-y / 2\right) d Y_{1} d X d y
\end{aligned}
$$

Use the Fourier inversion formula $\hat{\hat{\phi}}(x)=\phi(-x)$ to integrate over $y$ and $Y_{1}$, $J\left(a, f^{\prime}\right)$ equals:

$\gamma\left(a, \psi^{-1}\right)^{-1}|a|^{n-3 / 2} \int_{X \in F^{2 n-1}} \omega_{\psi^{-1}}\left(f^{\prime}\right) \phi_{0}\left(\frac{1-\langle a X, a X\rangle}{2 a}, a X, a\right) \psi\left(-X_{n}\right) d X$.

Make a change of variable $X \rightarrow a^{-1} X$, we get:

$\gamma\left(a, \psi^{-1}\right)^{-1}|a|^{-n-1 / 2} \int_{X \in F^{2 n-1}} \omega_{\psi^{-1}}\left(f^{\prime}\right) \phi_{0}\left(\frac{1-\langle X, X\rangle}{2 a}, X, a\right) \psi\left(-X_{n} / a\right) d X$.

The identity in Theorem 3 follows immediately from the above expression, Lemma 3 and the identity (13).

\section{References}

[Fl-Ma] Y. Flicker and J.G.M. Mars, Cusp forms on GSp(4) with SO(4) periods, preprint.

[F] M. Furusawa, On the theta lift from $S L_{2 n+1}$ to $\tilde{S p_{n}}$, J. Reine. Angew. Math., 466 (1995), 87-110.

[H] R. Howe, $\theta$-series and invariant theory, Proc. Symp. Pure. Math., 33(1) (1979), $275-285$.

[H2] Another look at the local $\theta$-correspondence for an unramified dual pair, in 'Israel Math. Conf. Proc.', 2 (1990), 93-124.

[J] H. Jacquet, On the nonvanishing of some L-functions, Proc. Indian Acad. Sci., 97 (1987), 117-155. 
[M-R] Z. Mao and S. Rallis, A trace formula for dual pairs, Duke Math J., 87(2), 321-341.

[Mo-V-W] C. Møglin, M.-F. Vignéras and J.-L. Waldspurger, Correspondances de Howe sur un corps p-adique, LNM 1291, Springer Verlag, 1987.

[PS-S] I. Piatetski-Shapiro and D. Soudry, On a correspondence of automorphic forms on orthogonal groups of order five, J. Math. Pures. Appl., 66 (1987), 407-436.

[R] S. Rallis, Langlands' functoriality and the Weil representation, Amer. J. Math., 104(3) (1982), 469-515.

[W] J.-L. Waldspurger, Demonstration d'une conjecture de duality de Howe dans le case $p$-adiques, $p \neq 2$, in 'Israel Math. Conf. Proc.', 2 (1990), 267-324.

[We] A. Weil, Sur certains groupes d'opérateurs unitaires, Acta Math., 111 (1964), 143-211.

[Z] D. Zinoviev, Relation of orbital integrals on $S O(5)$ and $P G L(2)$, Thesis, Ohio State University, 1997.

Received February 2, 1998 and revised February 10, 1999.

Rutgers UNIVERSity

NeWARK, NEWARK, NJ 07102

E-mail address: zmao@andromeda.rutgers.edu

The Ohio State University

Columbus, OH 43210

E-mail address: haar@math.ohio-state.edu 